\title{
Effect of the Periodontal Ligament of the Bilateral Support Teeth on the Stress Analysis of Dental Implant
}

\author{
Yanhua Xie, Xi Lu , Jianrun Zhang and Beibei Sun \\ School of Mechanical Engineering, the University of Southeast, Nanjing, China
}

\begin{abstract}
The aim of this work is to analyze the function of natural teeth's periodontal ligament and the effects of periodontal ligament on implants by the finite element method (FEM), when static functional loads occur. The finite element analysis models are established, which consist of fragment of mandible, natural teeth, periodontal ligament, and implant. Finite element software Abaqus is used to analyze the stress transfer in models with or without periodontal ligament, and mandibular first molar is implant. The implant obtained the maximum stress value of $87.71 \mathrm{MPa}$ when periodontal ligament of natural tooth is absent, but the maximum value reduces to $38.43 \mathrm{MPa}$ with the action of periodontal ligament. It illustrates that periodontal ligament has significant effects on stress transfer. When the finite element model of single natural tooth or dentition with implant is generated, periodontal ligament should be taken into account.
\end{abstract}

\section{Introduction}

Periodontal ligament can buffer chewing force and reduce the stress that spread to jaw bone, which has been extensively recognized [1]. The property of periodontal ligament has been researched continuously, and the values of elasticity modulus and poisson's ratio are various [2]. The linear elastic [3], bilinear elastic, nonlinear hyperelastic [4] or viscoelastic $[5,6]$ constitutive models have been extensively adopted to simulate the behaviour of the periodontal ligament. There are very few in vivo measurements have been performed. However, the specimens of the periodontal ligament were obtained from human dead bodies or animals [7]. There would be the inevitable errors and uncertainties of the values of periodontal ligament. Since the intensive and extensive investigation of the periodontal ligament is limited [2] and in vivo experiment is hardly carried out. It's difficult for us to obtain the accurate values.

There have been many studies about the effects of the positions, numbers [8] or geometrical parameters [9] on the dental implant, while the effects of the support of natural teeth on the side of denture were neglected [10-12] during the analysis of stress and deformation of implants. And in a lot of finite element models that stress of dental implant is analysed periodontal ligament is excluded.

In this paper, the specialized models with or without periodontal ligament are created and denture is supported by natural teeth, which are applied to evaluate and compare the values of the stress that transferred to the jaw bones under the same load.

\section{Materials and methods}

\subsection{Plan design}

For there is a variability in teeth and mandible bone morphology, the geometric model should be necessary to refer to the actual mandible shape and teeth morphology. The geometry of the model is produced with CT image slices of an adult human mandible obtained with a CT scanner. Scanned images are imported into the image segmenting and calculating software MIMICS (Materialise's interactive medical image control system). Based on the different values of Hounsfield of muscle, mandibular bone and teeth are segmented from the whole geometry image, and teeth are separated from alveolar bone in the same way. By performing calculate $3 \mathrm{D}$ order, the geometry models are reconstructed, while triangulated files are exported in STL format. Then, the files are inputted in the reverse engineering software Geomagic Studio 10. According to the measurement values of thickness of periodontal ligament from the faultage image, $0.2 \mathrm{~mm}$ is selected as the average thickness of the periodontal ligament.

When the dental crown is supported by the natural teeth, the stress distribution and transmission of the implant is analyzed.

In model 1, the two natural teeth that on the side of implant have periodontal ligament and support the crown of prosthesis. The finite element model consists of natural teeth with periodontal ligament, dental implant (mandibular first molars) and fragment of mandible as seen in Fig. 1.

In model 2 , the two natural teeth have no periodontal ligament and the finite element model includes natural teeth without periodontal ligament, dental implant 
(mandibular first molars) and fragment of mandible, which is shown in Fig. 2.
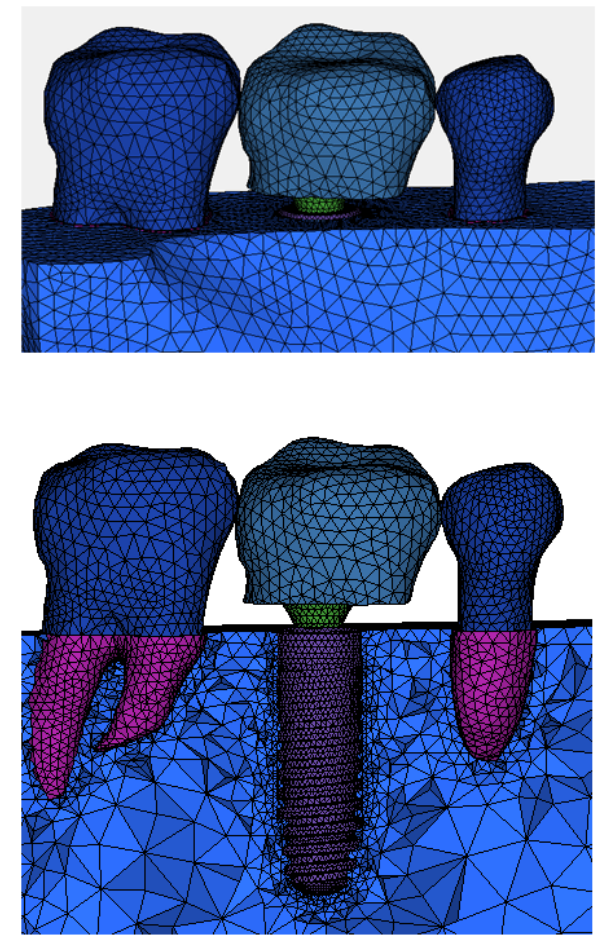

Figure 1. The finite element model with periodontal ligament.
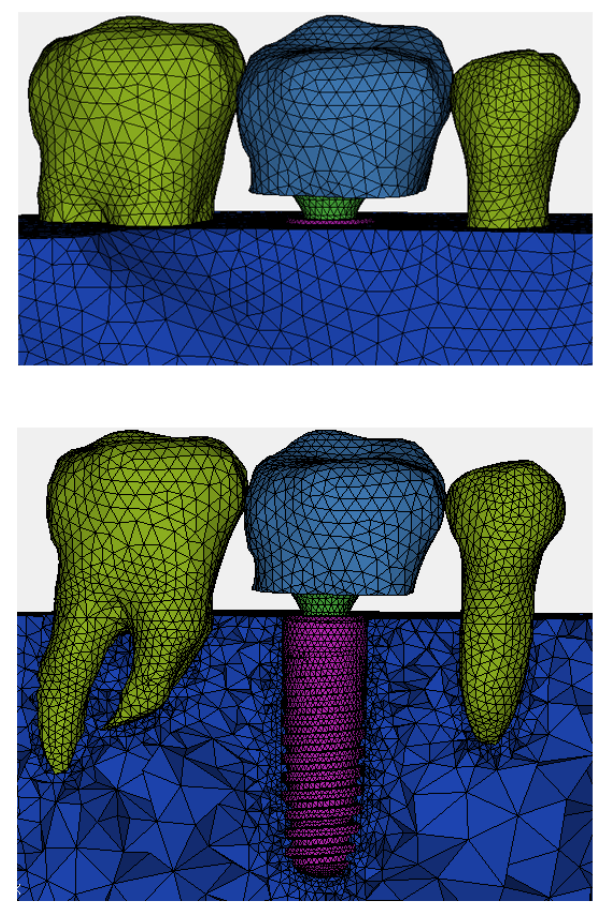

Figure 2. The finite element model without periodontal ligament.

\subsection{Material properties}

The materials of periodontal ligament, the second premolar (natural teeth), the second molars (natural teeth), titanium implants, mandible bone and metal-ceramic were considered continuous, homogeneous, isotropic linear elastic materials. Material properties are present in Table 1 [13-15].

Table 1. Material properties.

\begin{tabular}{|c|c|c|c|}
\hline Material & $\begin{array}{c}\text { Modulus of } \\
\text { elasticity } \\
(\mathrm{MPa})\end{array}$ & $\begin{array}{c}\text { Poisson's } \\
\text { ratio }\end{array}$ & $\begin{array}{c}\text { Density } \\
\left(\mathrm{g} / \mathrm{cm}^{3}\right)\end{array}$ \\
\hline natural teeth & 18600 & 0.31 & 3.1 \\
\hline $\begin{array}{c}\text { periodontal } \\
\text { ligament }\end{array}$ & 40 & 0.45 & 1.05 \\
\hline $\begin{array}{c}\text { mandible } \\
\text { bone }\end{array}$ & 13700 & 0.3 & 1.67 \\
\hline $\begin{array}{c}\text { titanium } \\
\text { implants }\end{array}$ & 110000 & 0.35 & 4.5 \\
\hline $\begin{array}{c}\text { metal- } \\
\text { ceramic }\end{array}$ & 84000 & 0.28 & 2.4 \\
\hline
\end{tabular}

\subsection{Boundary conditions}

In model 1, this section involves a total of nine contact pairs are generated in second premolar and internal surface of periodontal ligament, outside surface of periodontal ligament of the second premolar and the surface on mandible, the outside surface on second molar and internal surface of periodontal ligament, outside surface of periodontal ligament of the second molar and the surface on mandible, the surface on mandible and the surface on implants, the surface on implant and the surface on abutment, the surface on abutment and the inner surface on crown of molar, mesial surface of crown of molar and distal surface of second premolar, distal surface of crown of molar and mesial surface of second molar. Additionally, stresses of five surfaces that showed in Fig. 3 are analysed.

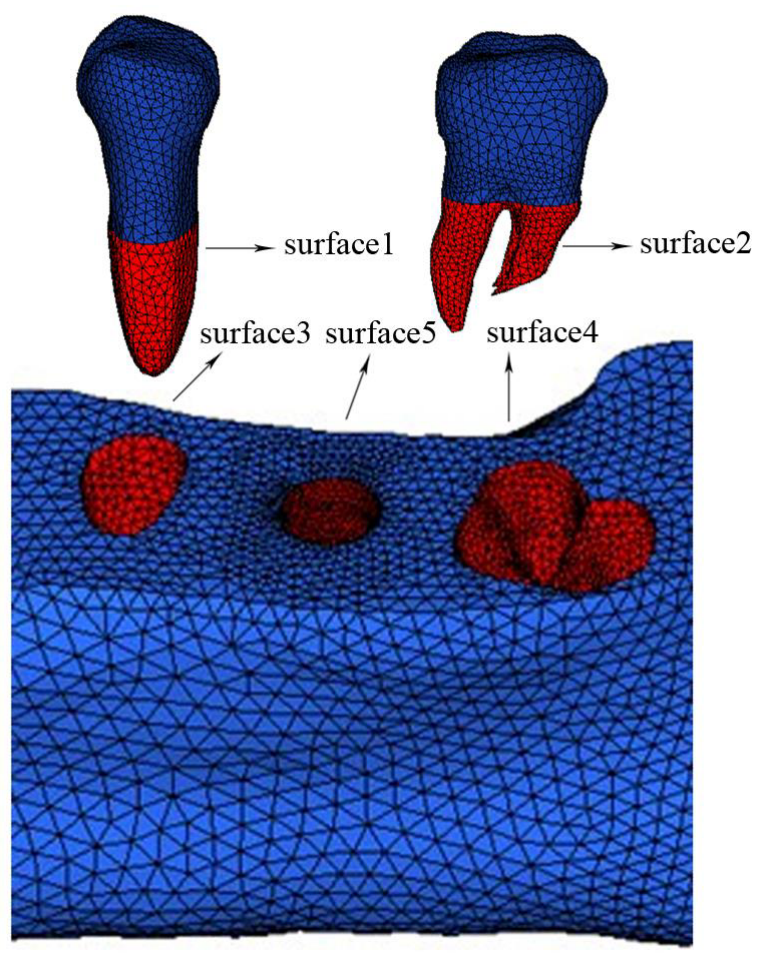

Figure 3. Five contact surfaces of model. 
Since the periodontal ligament is excluded in model 2, there are only seven contact pairs that between the outside surface of the second premolar and the surface on mandible, outside surface of the second molar and the surface on mandible, the surface on mandible and the surface on implants, the surface on implant and the surface on abutment, the surface on abutment and the inner surface on crown of molar, mesial surface of crown of molar and distal surface of second premolar, distal surface of crown of molar and mesial surface of second molar.

The contact surface of mandible and implant is assumed as Osseo integration and treated as tie constraint. The rest contact pairs keep close contact without gaps, and friction coefficients of 0.3 is considered for these contact surfaces.

The model 1 and model 2 are constrained at subjacent outer surface of mandible in all directions. The upper of the outer surface of is not exerted on any constraints.

Contact points are used to replace occlusal contact surface [16] and to simulate the surface to suffer from the load that perpendicular to the occlusal surface and located on the buccal cusp and tongue cusp of the teeth. Two contact points are selected on the second premolar. Four contact points are selected on the second molar. And four contact points are selected on crown on the top of the implant.

The value of applied force is $250 \mathrm{~N}$, which is regarded as the average force loading on each tooth [17, 18]. Boundary conditions and loads are shown in Fig. 4.

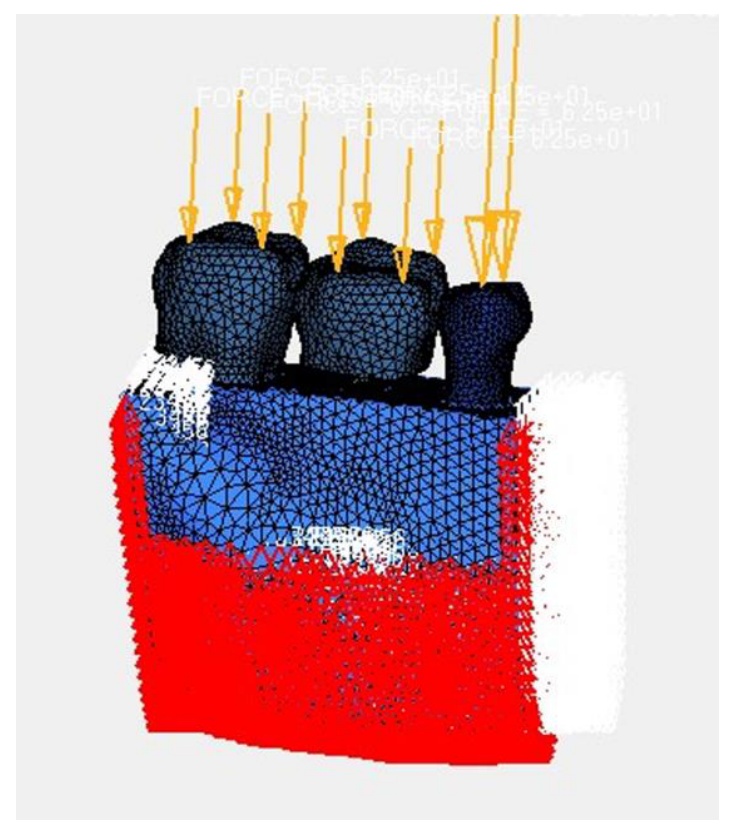

Figure 4. Forces and constraints.

\section{Results and discussion}

The maximum values of stress on the five surfaces and implant in model 1 are figured out and presented in Table 2. Additionally, the stress distributions on the five surfaces and implant in model 1 are shown in Fig. 5-7.
It can be seen that under the effect of the periodontal ligament the magnitude of stress on the surface on mandible is smaller than those on the root of the teeth.

On the position of second premolar, the maximum magnitudes of stresses on surface 1 and surface 3 are $9.45 \mathrm{MPa}$ and $2.27 \mathrm{MPa}$ respectively. In comparing the surface 1 and surface 3 , the maximum value of stress on the surface 1 is obviously higher than that in the surface 3 . And the value of stress on surface 3 declined 75.98 percent. On the position of second molar, the maximum magnitudes of stresses on surface 2 and surface 4 are $40.88 \mathrm{MPa}$ and $6.90 \mathrm{MPa}$. The maximum value of stress on surface 2 is many times greater than that on surface 4 . It's apparent that the values declined steeply almost 83.12 percent. Additionally, the maximum value of stress on surface 5 and implant are $13.28 \mathrm{MPa}$ and $38.43 \mathrm{MPa}$. The maximum value of stress on surface 5 is apparently decreased compare to that on the implant. And the values declined almost 83.12 percent.

As it shown in Fig 8, and the magnitude of stress on implant in the model 1 is $38.43 \mathrm{MPa}$, while that in the model 2 is $87.71 \mathrm{MPa}$. It's obvious that the value of the stress on implant in the model 1 is much less than that in the model 2. The model without the periodontal ligament would induce higher value of stress.

The present research is performed to analyse the function of natural teeth's periodontal ligament and the effects of periodontal ligament on implants. Two representative models are reproduced. Model 1 is used to simulating the biomechanical behaviour of implant under the condition that the nature teeth without periodontal ligament, while in the model 2 both the support teeth have the function of periodontal ligament. The analyses results adequately illustrate that periodontal ligament can buffer chewing force and reduce the stress [1].

Table 2. The values of stresses on the five surfaces

\begin{tabular}{|c|c|c|c|c|}
\hline $\begin{array}{c}\text { Upper } \\
\text { surface }\end{array}$ & $\begin{array}{c}\text { Maximum } \\
\text { stress(MPa) }\end{array}$ & $\begin{array}{c}\text { Lower } \\
\text { surface }\end{array}$ & $\begin{array}{c}\text { Maximum } \\
\text { stress(MPa) }\end{array}$ & $\begin{array}{c}\text { Proportion } \\
\text { of stress } \\
\text { decline }\end{array}$ \\
\hline surface1 & 9.45 & surface3 & 2.27 & $75.98 \%$ \\
\hline surface2 & 40.88 & surface4 & 6.90 & $83.12 \%$ \\
\hline implant & 38.43 & surface5 & 13.28 & $65.44 \%$ \\
\hline
\end{tabular}

Inaccurate results may result in the cases that the denture without the support of natural teeth on the side [10-12] during the analysis of stress and deformation of implants. Since the periodontal ligament is absent, the analysis results may much different.

The finite element model that included natural teeth with periodontal ligament is crucial in the biomechanical research of implant.

The magnitudes of the stresses on surfaces that located in alveolar bone are almost three times more than that on the root of teeth. And the maximum value of stress on implant in model 1 is up to $38.43 \mathrm{MPa}$. However, in comparing the maximum magnitude of stress on implant in the model 2 and that in the model 1, the value of the stress on implant in the model 2 is much higher. 


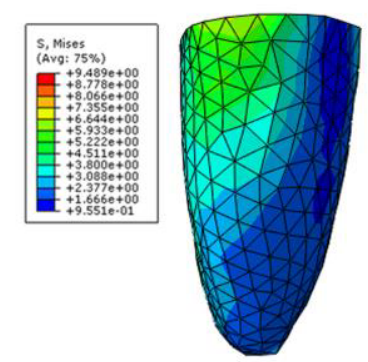

(a)

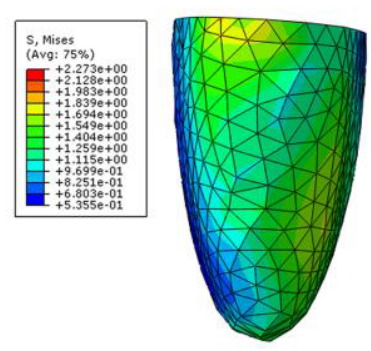

(b)
Figure 5. Stress distribution of the surfaces. (a) surface 1, (b) surface 3 .

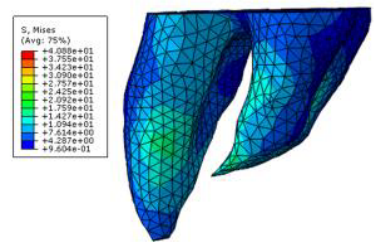

(a)

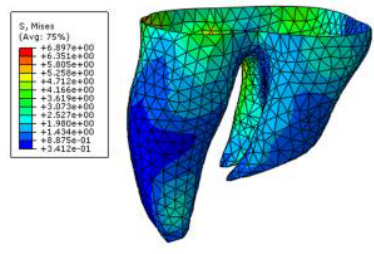

(b)
Figure 6. Stress distribution of the surfaces. (a) surface 2, (b) surface 4.

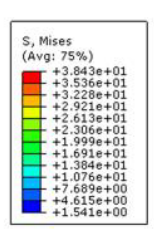

(a)

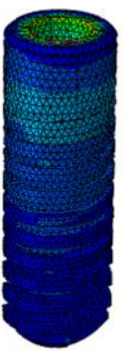

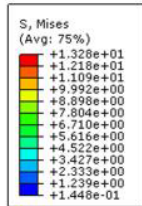

(b)

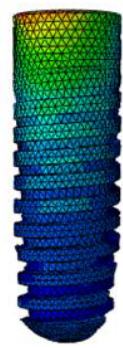

Figure 7. Stress distribution of the surfaces. (a) implant, (b) surface 5 .

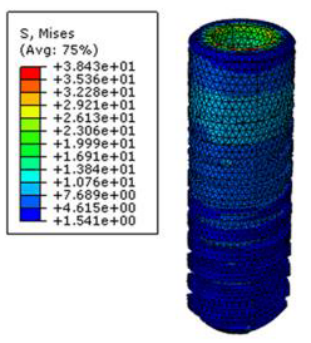

(a)

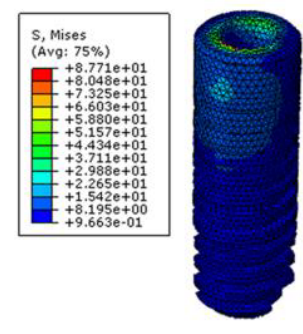

(b)
Figure 8. Stress distribution of the implants. (a) model 1, (b) model 2.

\section{Conclusions}

It clearly demonstrates that periodontal ligament has significant effect on stress transfer. The values of the stress of implant that with both side support of natural teeth will significantly increase from $38.43 \mathrm{MPa}$ to 87.71 MPa, when the periodontal ligament of natural teeth is not taken into consideration. It explicitly illustrates that the periodontal ligament of natural teeth that supporting the denture has significant influence on the stress analysis of the implant and should be take into consideration during the modelling.

\section{References}

1. M. Tunan, E. Sunbuloglu, E. Bozdag. J. B 47, $2883-$ 2890 (2014)

2. Xia Z, Jiang F, Chen J. A. J. O. D. O 4, 486-491 (2013)

3. I. A. V. P. Poiate, A. B. Vasconcellos, M. Mori. C. M. P. B 2, 292-299 (2011)

4. Qian L, Todo M, Morita Y. J. D. M 10, 1285-1292 (2009)

5. S. R. Toms, G. J. Dakin, J. E. Lemons. J. B 10, 14111415 (2002)

6. A. N. Natali, P. G. Pavan, C. Venturato, C. M. P. B 2, 193-198 (2011)

7. N. Yoshida, Y. Koga, C. L. Peng. M. E. P 8, 567-572 (2001)

8. T. Topkaya, M. Y. Solmaz. J. B 10, 2102-2109 (2015)

9. M. B. Toniollo, A. P. Macedo, R. C. S. Rodrigues. J. P. R 3, 206-212 (2013)

10. A. N. Natali, E. L. Carniel, P. G. Pavan. D. M 4, 561-569 (2008)

11. Lin D, Li Q, Li W. J. M. B. B. M, 5, 410-432 (2009)

12. L. Baggi, I. Cappelloni, F. Maceri. S. M. P. T 8, 971987 (2008)

13. P.M. Tulimar, B. Estevam. J. Med. Biol.Eng.Comp 48, 1107-1113 (2010)

14. T. Topkaya, M.Y. Solmaz. J. B 48, 2102-2109 (2015)

15. J.S. Ree, P.H. Jacobsen. J. B 18, 995-999 (1997)

16. Ding, X, et al. J. M. D 10, 168-175 (2010)

17. J. Middleton, M.L. Jones, J. Hiekman, etal. 10th comference of the European Society of Biomechanic, (1996)

18. G.G. Robert, M.P. John. Dental restoration materials science (2006) 